
Military Technical College
Kobry Elkobbah, Cairo, Egypt

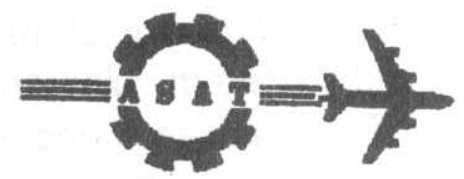

$8^{\text {th }}$ International Conference on Aerospace Sciences \& Aviation Technology

\title{
EXPERIMENTAL INVESTIGATION ON MISALIGNMENT EFFECT ON HYDRODYNAMIC TRANSMISSION PERFORMANCE
}

\author{
H. A. OMAR*, I. SALEH"*, S. KOSSA
}

\section{ABSTRACT}

The fluid coupling represents a form of fluid connectors that transmits the power by oil circulation through it, fluid coupling is one version of an integrated hydrodynamic drive. The performance of fluid coupling is affected by many sources of vibration; one of vibration sources is the misalignment between the input and output shafts of the fluid coupling. Assembling misalignment, usually results to a non uniform clearance distribution between pump and turbine stages of fluid coupling. This causes unsymmetric fluid forces on both stages; leading to fluid induced vibration. The amplitude of vibration may be greater than the allowable clearance between pump and turbine stages, that may cause solid contact between the stages and may provoke the mechanical failure of the transmission. This phenomenon of induced vibration, due to shafts misalignment of a fluid coupling has been investigated experimentally. The coupling stage was simulated by a rotating disk subjected to non-uniform jet forces applied on its rim. The results showed that; the higher the misalignment angle, the higher the vibration amplitude effect on the transmission performance. The effect of misalignment on the transmission performance increases at heavy duty loading. At the same misalignment angle; the higher the load, the higher the vibration amplitude effect on the transmission performance.

\section{KEY WORDS}

Mechanical engineering, Hydrodynamic Transmission, Vibration

\section{INTRODUCTION}

The performance of the hydrodynamic transmission suffers from many sources of vibration such as fluid induced vibration, misalignment that may occur between its input and output shafts, mistunning of rotor blade masses, incorrect assembly of runners

*Assistant, Dpt. of Mechanical Equipment, Military Technical College, Cairo, Egypt.

** Professor, Dpt. of Mechanical Power, Military Technical College, Cairo, Egypt.

Professor, Dpt. of AVCM , Miltary Technical College, Cairo, Egypt. 
Proceedings of the $8^{\text {th }}$ ASAT Conference, 4-6 May 1999

Paper FP-02

608

during the maintenance, as well as bad fixation of bearing and rotor shafts. Assembling misalignment, usually results to non uniform clearance distribution betweer pump and turbine stages of fluid coupling. This causes unbalance of fluid forces on both stages; leading to fluid induced vibration. A critical case would result in which vibration amplitude may exceed the clearance between coupling components. That may provoke the mechanical failure of the transmission. This phenomenon is investigated in this work.
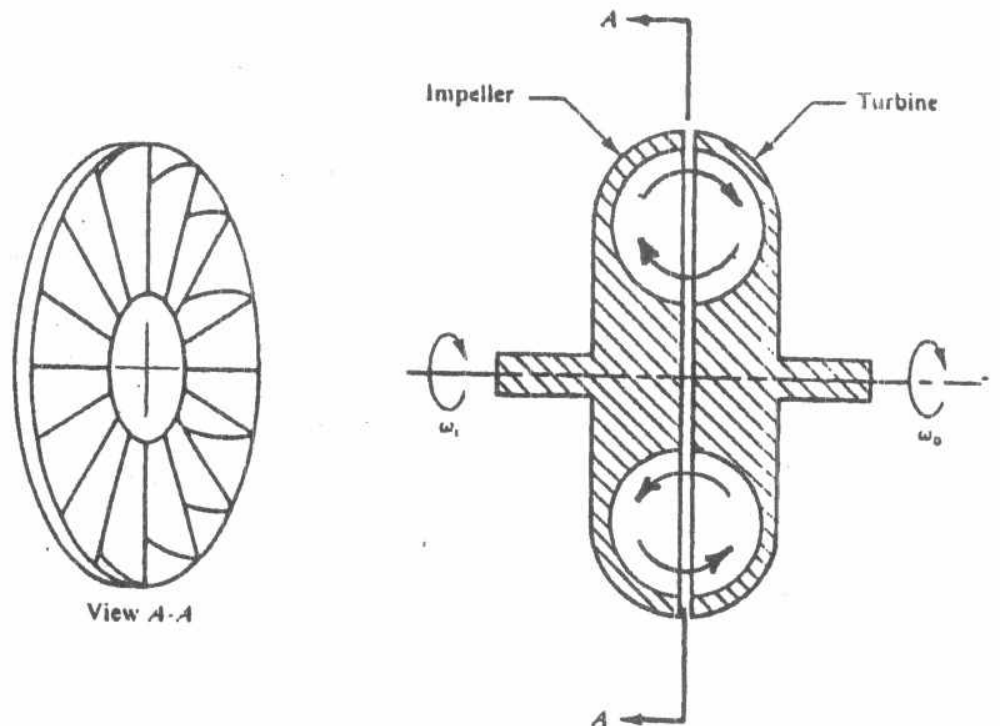

Fig.1. Fluid Coupling

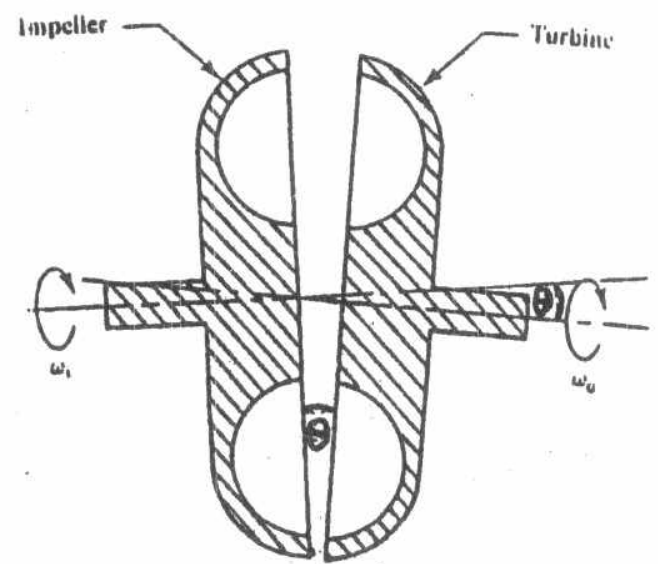

a. Schematic Diagram

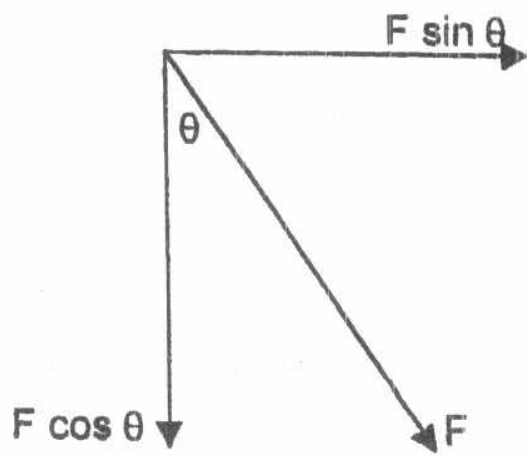

b. Effect Of Tilting Angle On Momentum Force.

Fig.2. Misalignment Between Fluid Coupling Runners 


\section{MISALIGNMENT OF WORKING SHAFTS}

"Perfect alignment " of the driving and driven shafts could not be achieved in practical application. Furthermore, if perfect alignment was achieved initially, it could not be maintained over an extended period due to machinery foundation, thermal expansion and contraction, as well as another similar effects. Therefore, a flexible connector suitable for transmitting rotary power without torsional slip and capable of accommodating unavoidable misalignment, has to be used. These connectors, in addition to transmitting power, may provide the following functions:-

1- permit easy assembly and disassembly of connected machines,

2- provide torsional vibration isolation and damping,

3- allow axial movement due to thermal expansion or contraction,

4- absorb axial movement to prevent thrust loading or to maintain part alignment,

5- permit angular, parallel or combination misalignment,

If the misalignment of a shaft is not reduced, through properly selected and aligned flexible connector, problems may arise, mainly:-

1- Noise.

2- Vibration.

3- Rapid wear of bearings and seals.

4- Gear damage or failure.

5- Shaft fatigue failure.

6- Coupling failure.

As discussed before, the misalignment phenomenon in fluid coupling could not be ignored. The types of misalignment are angular, parallel and a combination of angular and parallel. The misalignment between fluid coupling stages may cause the coupling failure due to non-uniform distribution of the momentum forces inside the fluid coupling. Also, the misalignment results in a vibration of the coupling stages; the vibration amplitude may be higher than the allowable clearance between the coupling stages causing the failure of
the system.

\section{SIMULATION OF FLUID COUPLING}

Fig.1. shows oil flow in a fluid coupling stage. Impeller shaft, or driving tourus, is driven by the engine and the turbine, or driven tourus, is mounted on the driven shaft. The transferred torque from the impeller to the turbine is due to changing in the fluid angular momentum, as fluid transfers to the turbine from the pump impeller. The misalignment effect may be viewed as coupling operation under non-uniform clearance between its stages, as shown in Fig.2. This will affect the momentum forces on coupling runners. To simulate this phenomenon, a spinning disk with constant angular velocity subjected to jet forces on its rim is used. The measurement of relative vibratory motion of a hydrodynamic transmission due to the effect of misalignment between the input and output shafts will be carried out by a simulation molding of one runner of hydrodynamic transmission. It is represented by a rotating disk of same outer diameter of a fluid coupling stage. The second runner is simulated by jet system. The unbalance fluid force will be simulated by applying non-uniform water jet. The jet are uniformly distributed on the outer rim of the 
disk Fig.3. The effect of misalignment between the fluid coupling runners is simulated by changing the angle of impinging jet system on the disk, that resulting in shanging of momentum force distribution on the disk rim.

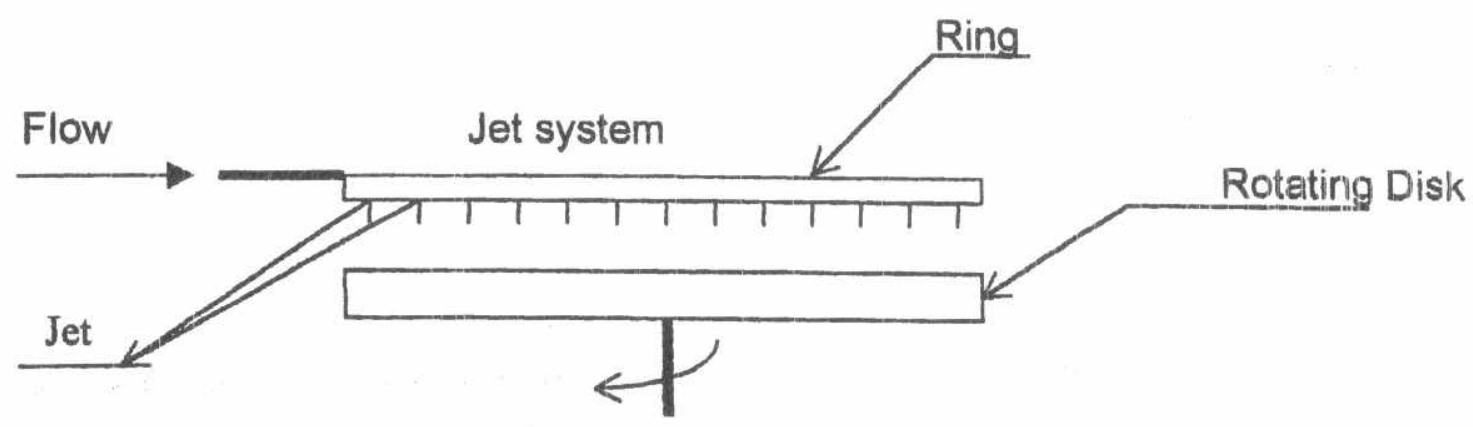

Fig.3. Rotating Disk With Impinging jet

\section{EXPERIMENTAL SET-UP}

In the real system the two runners of fluid coupling are located in a vertical plane, but in the simulation modeling the disk under an axial non uniform pressure is located in a horizontal plane to eliminate the effect of the disk weight as a radial force component. Hence the task is to determine the disk vibration characteristics under the effect of axial jet force. The vibration phenomenon is investigated by using five strain gauges bonded radialy, on maximum strain area, at angle equals 72 degree with respect to each other. As shown in Fig.4. a rotating circular disk is coupled to the driving motor shaft.. Forty four impinging jet were uniformly distributed by a circular ring over the circumfrential of the disk. A hydraulic pump would discharge a fluid (water) to the impinging jets through a pressure hose. The inlet jet pressure is controlled via a control valve and the misalignment effect is verified by tilting the circular ring to change the uniformity of the momentum force.

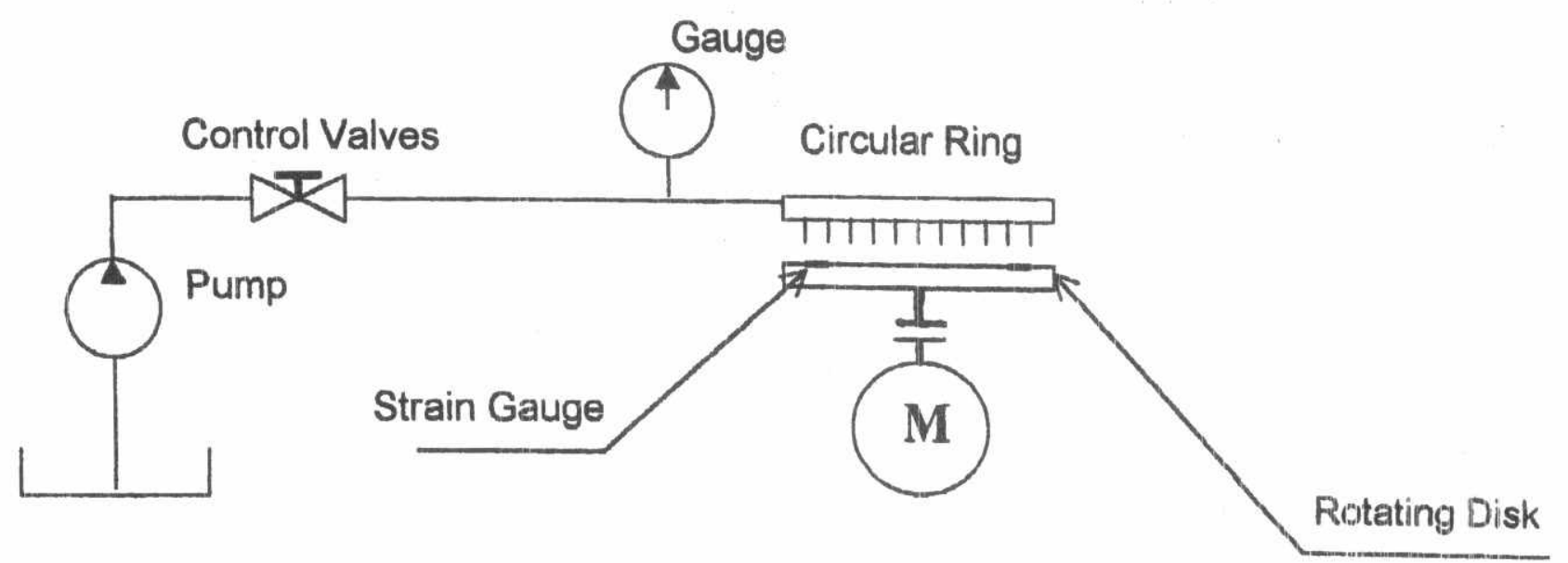

Fig.4. Block Diagram of Test Rig. 
The misalignment phenomenon which results in a vibration of hydrodynamic transmission has been investigated by measuring the strain distribution on a rotating disk. The measurements were carried out for different angles of the misaligned load. The strain distribution on the outer rim of the disk gives an indication about the vibration amplitude. In other words, a high strain at a point means a high vibration amplitude at this point. Measurements were carried out for strain distribution due to:-

1- The free rotation effect ( under the effect of centrifugal force. )

2- The effect of fluid momentum force, that exists in horizontal plane.

3- The effect of fluid momentum force on oblique plane for misaligned angles 3,6 and 9 degrees and for jet inlet pressure $p=1.2,1.4,1.6$ and 1.8 bar.

To increase the accuracy, the measurements were repeated five times. The average values of these five readings is considered.

Calibration of the system has been carried out before measurement of the strain distribution of the model due to the effect of the load.

\section{CALIBRATION OF THE MEASURING TRANSDUCERS}

Five strain gauges are distributed uniformly on the disk and they were bonded at angle 72 deg. with respect to each other. The measuring transducer was calibrated within putting standard weights on the disk rim ( the disk is static ). The forces used in calibration range from 1 to $10 \mathrm{~N}$; the response of the transducer is measured by $1526 \mathrm{HBM}$ amplifier.

Fig.5. illustrates the relation between the load and measured strain (load against the strain readings ).

\section{CALIBRATION OF THE JET SYSTEM}

The calibration of the jet system was carried out by measuring a specified discharge rate for each jet at a specified pressure. The measured data are represented in Fig.6, the $x$ axis represents the number of jet and the $y$-axis represents the discharge rate; it is showed that the uniformity of momentum force is verified. 


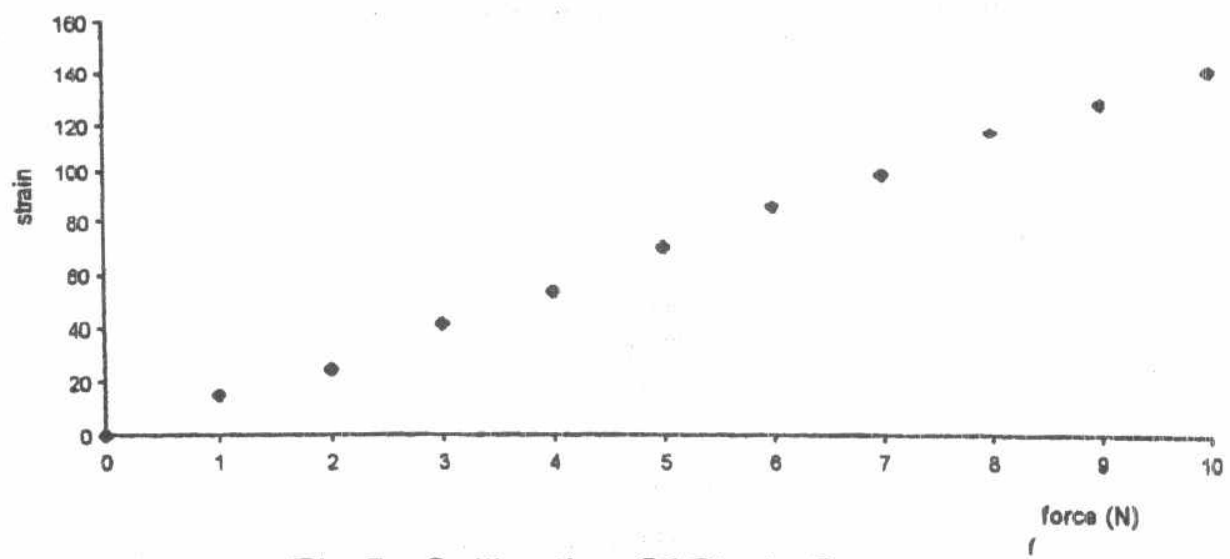

Fig.5. Calibration Of Strain Gauges

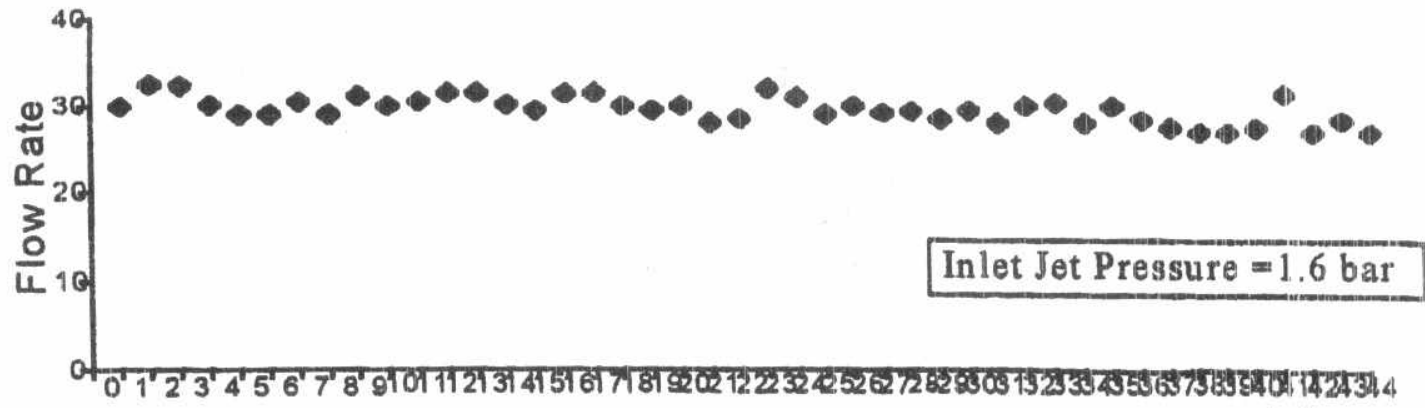

jet no.

Fig.6. Calibration of water jet system

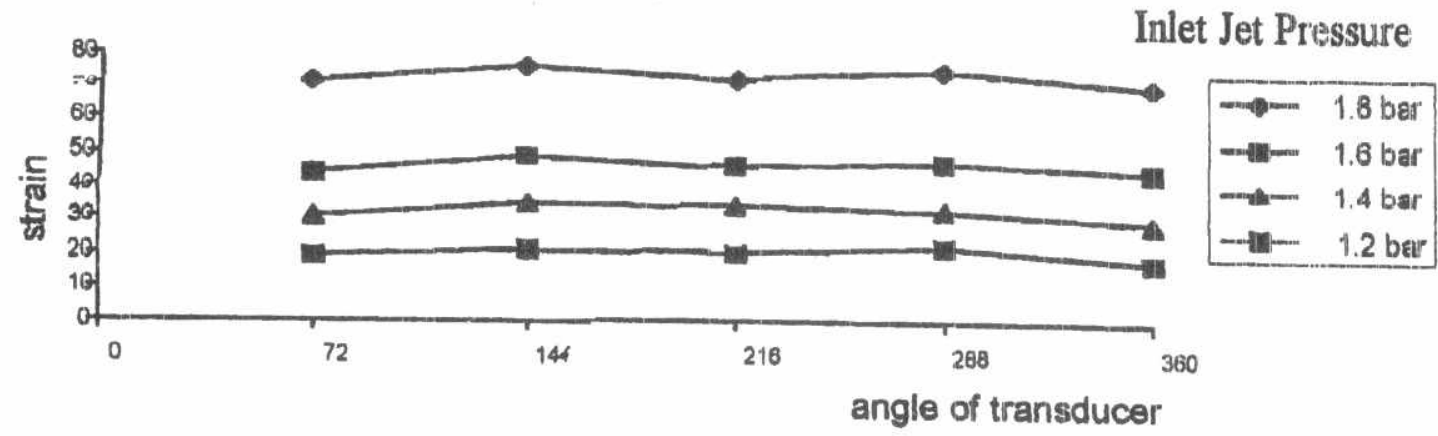

FIG.7. Strain level for different inlet jet pressure at zero tilted angle 


\section{EXPERIMENTAL RESULTS}

Fig.7. shows the strain distribution on the rotating disk under the effect of a different values of uniform momentum forces, the $y$-axis represents the strain readings and $x$-axis represents the distribution of the transducers on the disk; i.e the first strain gauge exists at angle $72^{\circ}$ and the second one exists at angle $144^{\circ}$. Results showed that there is a slight difference between the strain readings for each load, that is due to an allowable error in measuring system. A good agreement between the results could be achieved due to the effect of rotation of the disk at a constant speed and due to the uniform distribution of the jet over the rotating disk. The strain is found to be increased with increasing the momentum forces, the effect of changing the distribution of the momentum forces over the rotating disk on the level of strain results will be discussed in the following section. Fig. 8 . to Fig.10. illustrate the effect of misalignment angles on the rotating disk at various values of momentum forces. In Fig.8. the variation of measured data at specified points is noticed at misalignment angle equals 3 degree, and peak values are existed due to the effect of misalignment of the load at different values of momentum forces.

In Fig.9. the misalignment angle increased to be 6 degree, so the variation in measuring data is higher than which observed in Fig.8. In Fig.10. the misalignment angle equals 9 degree, therefore more variation of the measured data is observed, where at higher degree of misalignment a great difference between the maximum peaks and minimum peaks is noticed, that led to increase the vibration effect on the rotating disk.

Fig.11. to Fig.13. illustrate the effect of the load on the rotating disk at various values of misalignment angles. In Fig.11. the maximum amplitudes of the strain distribution are noticed for different misalignment angles, ( 9,6,3 degree ) at inlet jet pressure equals 1.8 bar. In Fig.12. the maximum amplitudes of the strain distribution are also noticed for different misalignment angles, $(9,6,3$ degree $)$ but its values are less than that observed in Fig.11. since the value of inlet jet pressure is lowered to 1.6 bar. In Fig.13. the maximum amplitudes are less than that observed in Fig.11. and Fig.12. where the value of inlet jet pressure is lowered to be 1.4 bar. These amplitudes led to increase the vibration of the disk. 


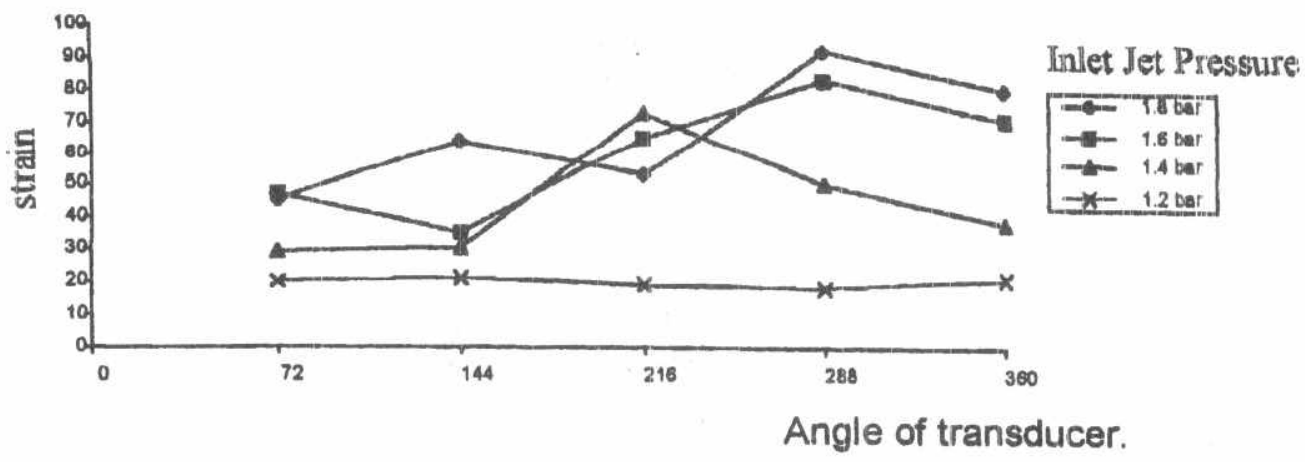

FIG.8. Strain Distribution At Misaligned Angle 3 Deg.

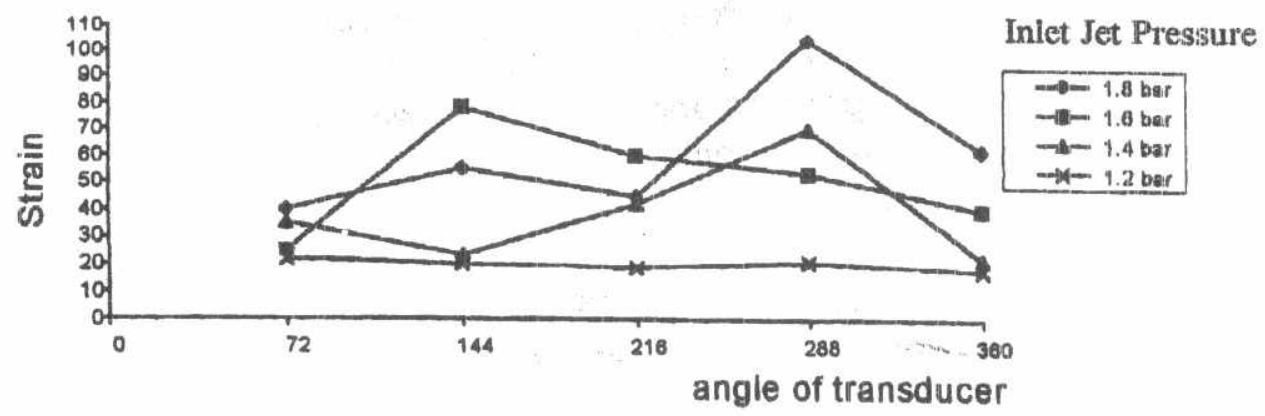

FIG.9. Strain Distribution At Misaligned Angle 6 Deg

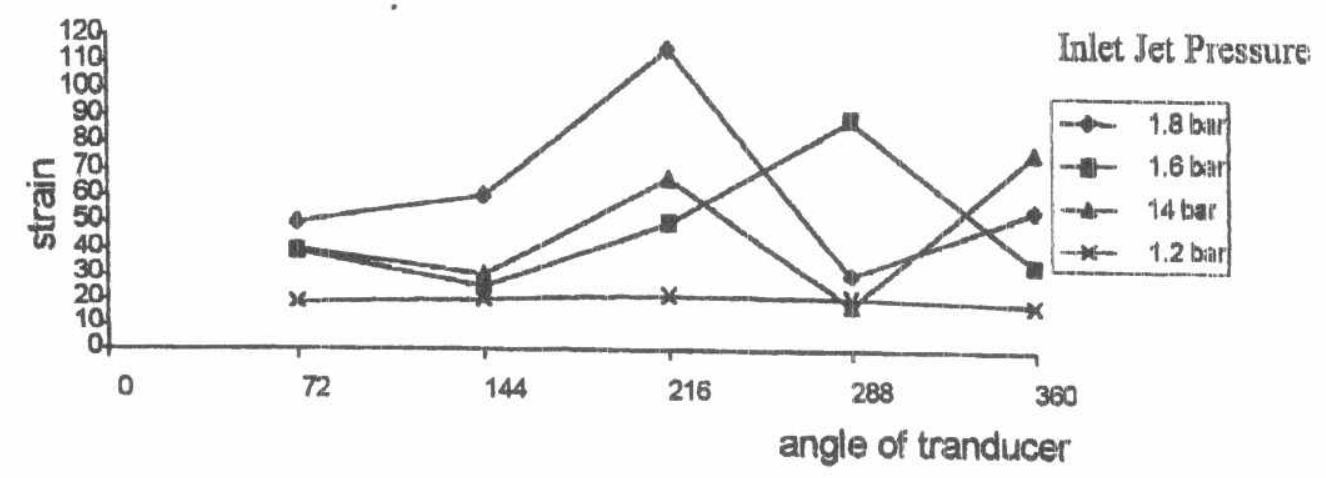

FIG.10. Strain Distribution At Misaligned Angle 9 Deg 


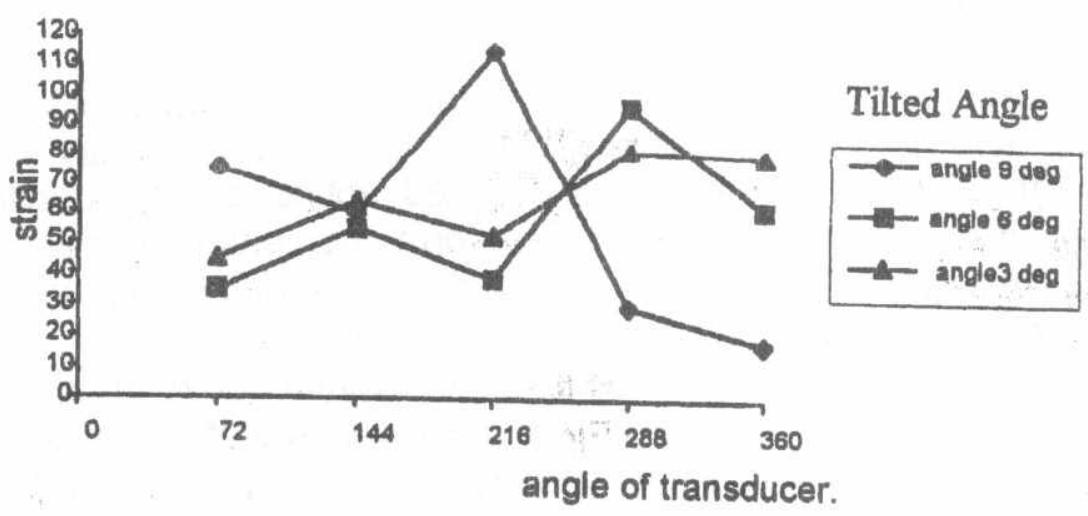

FIG.11. Strain Distribution At Inlet Jet Pressure 1.8 bar.

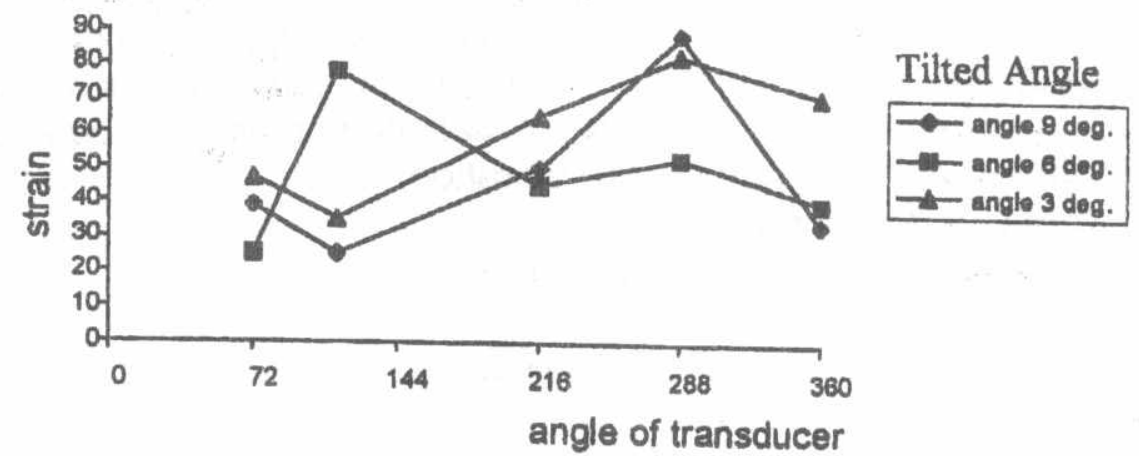

FIG.12. Strain Distribution At Inlet Jet Pressure 1.6 bar.

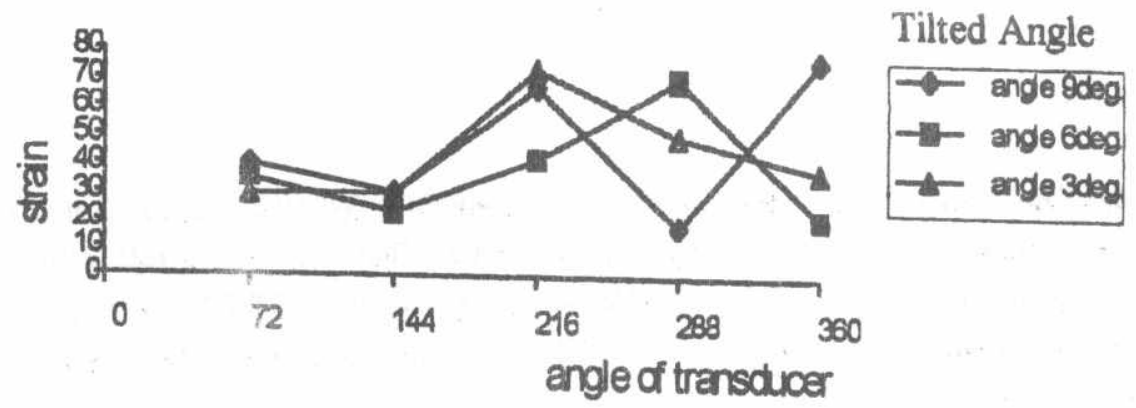

FIG.13. Strain Distribution At Inlet Jet Pressure 1.4 bar. 


\section{ANALYSIS OF RESULTS}

In Fig.7. the relation between the strain readings and transducers location is nearly uniform, this may be attributed to a rotation of the disk at a constant speed, and due to an uniform centrifugal forces of the disk masses acting on the transducers, tending to stretching the disk. Also the effect of uniform momentum forces on the strain level distribution is nearly uniform. This may be due to a-uniform distribution of the jet as well as uniform distribution of the momentum forces on the disk.

Fig.8. to Fig.10. showed the effect of tilted jet system above the disk for different values of inlet jet pressure. And Fig.11. to Fig.13. showed the effect of inlet jet pressure on the rotating disk for different values of misalignment angles. The maximum amplitudes that observed in Fig.8. to Fig.13. were existed due to the misalignment angles effect. That will result in varying the normal forces direction acting on the disk rim and hence variation of the strain level distribution. The tilted momentum forces would be resolved to two components, one of them causes the radial strain which is a function in vibration amplitudes and equals to the value of the momentum force times $\cos \theta$, as shown in Fig.2.b. ( where $\theta$ is the angle of misalignment ). The variation of the load on the disk rim resulting in varying the vibration amplitude at different points according to the applied load. With increasing the tilting angle (misalignment angle $\theta$ ), a part of jet would have a lower load causing lower vibration amplitude at some points while causing higher amplitude at another points. As the misalignment angle increases, the maximum amplitude increases, and causing severe vibration.

* For jet inlet pressure equal 1.8 bar;

- at $\theta=9$ deg., the maximum amplitude is 85 strain.

- at $\theta=6$ deg., the maximum amplitude is 62 strain.

- at $\theta=3$ deg., the maximum amplitude is 47 strain.

* For jet inlet pressure equal $1.6 \mathrm{bar}$;

- at $\theta=: 9$ deg., the maximum amplitude is 64 strain.

- at $\theta=6$ deg., the maximum amplitude is 53 strain.

- at $\theta=3$ deg., the maximum amplitude is 48 strain.

* For jet inlet pressure equal 1.4 bar;

- at $\theta=9$ deg., the maximum amplitude is 60 strain.

- at $\theta=6$ deg., the maximum amplitude is 48 strain.

- at $\theta=3$ deg., the maximum amplitude is 45 strain.

\section{CONCLUSION}

The misalignment between the coupling runners would lead to increase the allowable clearance between the fluid coupling runners, that would cause a solid contact between the impeller and turbine. This phenomena could cause the failure of the fluid coupling. Experimental investigation of the misalignment showed (for diameter $=31 \mathrm{~cm}$. and speed $=1500$ r.p.m.) that :-

1- The misalignment between hydrodynamic transmission runners is a source of vibrations that would affect on its performance.

2- The higher the misalignment angle, the higher the vibration effiect on the transmission performance. 
3- The effect of the misalignment on the transmission performance increases at heavy loading.

4- At the same misalignment angle; the higher the load, the higher the vibration amplitude effect on the transmission performance.

\section{RECOMMENDATION}

The assembling of the hydrodynamic transmission must be carried out with high accuracy level to avoid the misalignment effect.

\section{REFERENCES}

[1] Childs, "Fluid-Structure Interaction Forces At Pump-Impeller-Shroud Surfaces For Axial Vibration Analysis, " Transaction of ASME, vol. 113, pp 108-114, (1991).

[2] W. Childs, and p. williams, "Influence of Impeller shroud Forces on Turbpump Rotor Dynamics," Transaction of ASME, vol. 113, pp 508-515, ASME(1991).

[3] Geuelich, and U. Bbolleter, "Pressure Pulsation in Centrifugal Pumps," Transactions of ASME, vol. 114, pp 272-279, (1992).

[4] L. Jay, and D. W. Burns, "Characteristics of The Diameteral Resonant Response of a Shrouded Fan Under a Prescribed Distortion," Journal of Vibration, Acoustics, Stress, and Reliability in Design, vol. 108, pp125-131, (1986).

[5] V Srinivasan, and D. G. Cutts, "Measurement of Relative Vibratory Motion at The Shroud Interfaces of a Fan," Journal of Vibration, Acoustics, Stress, and Reliability in Design,vol. 106, pp 189-197, (1984).

[6] L. Deweel, and L. D. Mrtchell, "Detection of a Misaligned Disk Coupling Using Spectrum Analysis," Journal of Vibration, Acoustics, Stress, and Reliability in Design, vol. 106, pp 9-16, (1984).

[7] Barasch, and Y. Chen, "On The Vibration of a rotating Disk," Journal of Applied Mechanics, pp1143-1144, december (1972).

[8] H. Omar, "Effect of Misalignment on Hydrodynamic Transmission Performance," Msc. Thesis, Military Technical College, Cairo, ( 1997 ). 